\title{
Phosphorylation of islet-1 serine 269 by CDK1 increases its transcriptional activity and promotes cell proliferation in gastric cancer
}

\author{
Qiong Shi ${ }^{1}$, Xiaomei $\mathrm{Ni}^{1}$, Ming Lei ${ }^{1}$, Quansong Xia ${ }^{1}$, Yan Dong ${ }^{2}$, Qiao Zhang ${ }^{3}$ and Weiping Wang ${ }^{4^{*}}$ (])
}

\begin{abstract}
Background: Despite recent advances in diagnostic and therapeutic approaches for gastric cancer (GC), the survival of patients with advanced GC remains very low. Islet-1 (ISL1) is a LIM-homeodomain transcription factor, which is upregulated and promotes cell proliferation in GC. The exact mechanism by which ISL1 influences GC development is unclear.

Methods: Co-immunoprecipitation (co-IP) and glutathione S-transferase (GST)-pulldown assays were employed to evaluate the interaction of ISL1 with CDK1. Western blot and immunohistochemistry analyses were performed to evaluate the ability of CDK1 to phosphorylate ISL1 at Ser 269 in GC cell and tissue specimens. Chromatin immunoprecipitation (ChIP), ChIP re-IP, luciferase reporter, and CCK-8 assays were combined with flow cytometry cell cycle analysis to detect the transactivation potency of ISL1-S269-p and its ability to promote cell proliferation. The self-stability and interaction with CDK1 of ISL1-S269-p were also determined.
\end{abstract}

Results: ISL1 is phosphorylated by CDK1 at serine 269 (S269) in vivo. Phosphorylation of ISL1 by CDK1 on serine 269 strengthened its binding on the cyclin B1 and cyclin B2 promoters and increased its transcriptional activity in GC. Furthermore, CDK1-dependent phosphorylation of ISL1 correlated positively with ISL1 protein self-stability in NIH3T3 cells.

Conclusions: ISL1-S269-p increased ISL1 transcriptional activity and self-stability while binding to the cyclinB1 and cyclinB2 promoters promotes cell proliferation. ISL1-S269-p is therefore crucial for tumorigenesis and potentially a direct therapeutic target for GC.

Keywords: ISL1, Phosphorylation, CDK1, Transcription activity, Gastric cancer

\section{Introduction}

Gastric cancer (GC) is the fifth most common malignancy and the third leading cause of cancer-related deaths(Ferlay et al. 2015). China is among the countries

\footnotetext{
*Correspondence: wwp@bjmu.edu.cn

${ }^{4}$ Department of Biochemistry and Molecular Biology, School of Basic Medical Sciences, Beijing Key Laboratory of Protein Posttranslational Modifications and Cell Function, Key Laboratory of Molecular

Cardiovascular Sciences, Ministry of Education of China, Peking University, Beijing, P. R. China

Full list of author information is available at the end of the article
}

with the highest incidence of GC, accounting for over $40 \%$ of all new cases of GC worldwide(Bu and Ji 2013). Despite recent advances in diagnostic modalities and the development of molecular-targeted drugs, the survival rates of patients with advanced $\mathrm{GC}$ remains very low(Waddell et al. 2013). Understanding the precise molecular mechanisms underlying GC progression is therefore crucial for the development of effective therapeutic strategies against GC.

ISL1 or human insulin gene enhancer binding protein 1 (islet-1) is a LIM homeodomain transcription factor encoded by the ISL1 gene(Karlsson et al. 1990), and original author(s) and the source, provide a link to the Creative Commons licence, and indicate if changes were made. The images or other third party material in this article are included in the article's Creative Commons licence, unless indicated otherwise in a credit line to the material. If material is not included in the article's Creative Commons licence and your intended use is not permitted by statutory regulation or exceeds the permitted use, you will need to obtain permission directly from the copyright holder. To view a copy of this licence, visit http://creativecommons.org/licenses/by/4.0/. 
functions as a transcription factor that regulates the expression of genes involved in heart, motor neuron, and pancreas development and foregut morphogenesis(Bu et al. 2009; Guo et al. 2011; Kim et al. 2019; Shi et al. 2009; Zhang et al. 2018). ISL1 is a marker for pancreatic neuroendocrine tumors and their metastases(Dong et al. 1991; Wang et al. 2014). We previously reported that ISL1 is highly expressed in GC and is correlated with advanced tumor-nodes-metastasis (TNM) stage, lymph node metastasis, and poorer overall survival(Guo et al. 2015). Further, ISL1 could activate cyclinB1, cyclinB2, and c-myc expression in GC cells by directly binding to conserved binding sites on their promoter or enhancer, and thereby promote GC cell proliferation(Shi et al. 2016). However, the exact mechanisms by which ISL1 modulates GC development remain unknown. Understanding the roles of this protein in GC development and the ways in which it could be modulated could be of great research and clinical interest.

Post-translational phosphorylation plays critical roles in the assembly of signaling and repair proteins in several biological progresses (Sharma et al. 2014). Although several phosphorylation sites such as T182 (Zahedi et al. 2008) and S221(Mertins et al. 2013; Sharma et al. 2014) have been identified in ISL1 proteins, but the molecular mechanisms that govern their physiological effects remain unclear. Further, ISL1 overexpression or knockdown leads to increased or decreased GC cell proliferation, respectively, and can influence cell cycle transition (Guo et al. 2019; Shi et al. 2016). CDK1 is a major cell cycle transition kinase (McDonald et al. 2020); we therefore investigated whether ISL1 is regulated by CDK1.

In this study, we aimed to elucidate the molecular interactions of endogenous ISL1 with CDK1 in GC cell lines. Our results showed that CDK1 phosphorylated ISL1 Ser-269, which promoted its transcriptional activity and increased protein self-stability. The $\mathrm{S}^{269-}$ ISL1-CDK1 complex increased the proliferation in GC cells. Collectively, our results provide the first evidence that CDK1 phosphorylates ISL1 Ser-269, and provides insights into the role of ISL1 in GC.

\section{Methods and materials}

\section{Cell lines and culture}

The GC cell line BGC823 (ICLC: HTL98007), was purchased from Instituto Nazionale per la Ricerca sul Cancro (ICLC, Genova, Italy). MGC803, MKN28, and an immortalized human gastric epithelial cell line, GES1, were gifts from Profs. Shou CC and Ke Y (Peking University Cancer Hospital). Cells were cultured in Dulbecco's modified Eagle's medium (DMEM) supplemented with $10 \%$ fetal bovine serum (FBS), $80 \mathrm{U} / \mathrm{ml}$ penicillin, and $100 \mathrm{mg} / \mathrm{ml}$ streptomycin at $37^{\circ} \mathrm{C}$ under $5 \% \mathrm{CO}_{2}$.

\section{Immunoprecipitation and western blot analysis}

Cell lysates were prepared using radioimmunoprecipitation assay (RIPA) lysis buffer (P0013E; Beyotime, Shanghai, China) supplemented with protease inhibitor cocktail (469,313,200; Roche, Basle, Switzerland). Immunoprecipitation and western blot analysis were carried out as described previously (Liu et al. 2009), with ISL1 for Co-IP (H00003670-M05, Abnova, Taipei, China) and ISL1 for western blotting (ab109517, Abcam). Monoclonal anti-CDK1 (\#9116), GAPDH (\#2118), rabbit polyclonal anti-CDK1-T161P (\#9114), and mouse monoclonal anticyclin B1 (\#4135) were purchased from Cell Signaling Technology (Danvers, MA, USA). Mouse monoclonal anti-phosphoserine (05-1000) was obtained from Merck Millipore (Millipore Corp, Billerica, MA, USA). Rabbit polyclonal antibody against the phosphorylation site of ISL1 at Ser 269 (ISL1-S269) (1:1,000) was produced with the synthetic phosphopeptide MTGTPMVAA[Sp]PERHDGGLQ. (Absin Bioscience Inc., Shanghai, China)

\section{Glutathione S-transferase (GST) pull-down assay}

GST fused with full-length ISL-1 or various truncated ISL-1 proteins were prepared as described previously (Zhang et al. 2009). Biotin-labeled wild-type CDK1 was synthesized using the TNT Quick Coupled Transcription/Translation System (L5020, Promega, Madison, WI, USA) (Wang et al. 2016).

\section{Immunohistochemistry (IHC)}

All human GC tissue specimens (35 poorly differentiated adenocarcinoma, 22 moderately differentiated adenocarcinoma, 3 well-differentiated adenocarcinoma) were obtained from the Pathology Department, School of Clinical Oncology, Kunming Medical University (China). IHC was performed on 5-mm-thick serial sections of formaldehyde-fixed, paraffin-embedded tissue blocks derived from GC with paired adjacent tissue. The sections were incubated overnight at $4{ }^{\circ} \mathrm{C}$ with an antibody against ISL1 phosphorylated at Ser 269 (ISL1-S269-p) $(1: 150)$ produced using the synthetic phosphopeptide MTGTPMVAA[Sp]PERHDGGLQ (Absin Bioscience Inc, Shanghai, China). The extent of staining was defined as follows: 0\%: -, 1-24\%: +, 25-49\%: ++, 50-74\%: +++, and $75-100 \%:++++$.

\section{Chromatin immunoprecipitation (ChIP) and ChIP re-IP assays}

ChIP and ChIP re-IP experiments were performed in MKN28 and MGC803 cells as previously described(Zhang et al. 2007). After elution, real-time PCR or conventional PCR were performed to amplify the 
Table 1 Primer sequences

\begin{tabular}{ll}
\hline Gene & Primer sequences $\left(\mathbf{5}^{\prime} \mathbf{-} \mathbf{3}^{\mathbf{\prime}} \mathbf{)}\right.$ \\
\hline CCNB1 & Sense: CCGCTTCGGACTGCGAACTAA \\
& Antisense: AGAGCAGGCAGCAGCTAAGAAGG \\
CCNB2 & Sense: GCGGTATTGAATCCTGGAACAAG \\
& Antisense: CGGACTGAAAAGGGAGGACACT \\
\hline
\end{tabular}

Table 2 RNAi duplex sequences

\begin{tabular}{ll}
\hline Gene & RNAi duplex Sequences (5'-3) \\
\hline CDK1-1 & Sense: CCAUGGAUCUGAAGAAAUA dTdT \\
CDK1-2 & Antisense: dTdT GGUACCUAGACUUCUUUAU \\
& Sense: GCCAGAAGUGGAUCUUUA dTdT \\
CDK1-3 & Antisense: dTdT CGGUCUUCACCUUAGAAAU \\
& Sense: UCGGGAAAUUUCUCUAUUA dTdT \\
& Antisense: dTdT AGCCCUUUAAAGAGAUAU \\
& Antisense: ACGUGACACGUUCGGAGAAT \\
\hline
\end{tabular}

DNA fragment containing the CCNB1 or CCNB2 promoters, covering the ISL1/CDK1 binding sites, with the primers listed in Table 1.

\section{Plasmid transfection and RNA interference}

Cells were transfected with plasmids or synthesized siRNA for gene overexpression or knockdown, respectively. The plasmid constructs pcDNA3.1-ISL1, control pcDNA3.1, pLL3.7-ISL1-siRNA (si-ISL1), and control pLL3.7-Non-silencer have been previously described. (Guo et al. 2011). The pcDNA3.1-ISL1-S269A plasmid was constructed by TransGen Biotech (Beijing, China). RNAi duplexes for CDK1 (si-CDK1) and control siRNA (non-silencing, Non) were obtained from GeneChem (Shanghai, China; Table 2) DNA plasmids encoding CDK1 (Addgene plasmid \#1886) and CDK1-DN (Addgene plasmid \#1887) were obtained from Addgene. Site-directed mutagenesis was commercially performed by GeneChem (Beijing, China), and Ser 269 in ISL1 was replaced with alanine. Transfection of the plasmids and siRNA was carried out using LipofectamineTM2000 (Invitrogen, Carlsbad, CA, USA) according to the manufacturer's protocols. After $48 \mathrm{~h}$ of transfection, cells were harvested. All transfections were performed at least three times.

\section{Luciferase assay}

Plasmid transfection and luciferase activity detection were performed as described previously (Liu et al. 2011). The cyclinB1-luc and cyclinB2-luc reporters were constructed by TransGen Biotech (Beijing, China).

\section{Cell proliferation assays}

The cell proliferation was assayed using CCK-8 as described previously (Guo et al. 2011).

\section{Flow cytometry cell cycle analysis}

For cell cycle analysis, cells were fixed in ice-cold $70 \%$ ethanol and incubated at $4{ }^{\circ} \mathrm{C}$ overnight. Fixed cells were washed with phosphate-buffered saline (PBS) and treated with $100 \mu \mathrm{g} / \mathrm{ml}$ RNase A at $37^{\circ} \mathrm{C}$ for $20 \mathrm{~min}$. After staining with propidium iodide $(50 \mu \mathrm{g} / \mathrm{ml})$, the cells were subjected to fluorescence-activated cell sorting (FACS) on a FACScan instrument (Becton Dickinson, Franklin Lakes, NJ, USA). Cell debris and fixation artifacts were gated out and cell populations at the $G_{0} / G_{1}, S$, and $G_{2} / M$ phases were quantified using the ModiFit LT v2.0 software.

\section{Statistical analysis}

Data are expressed as the mean \pm standard deviation (SD). All statistical analyses were conducted using SPSS (version 20; SPSS, Chicago, Illinois). $\chi^{2}$ tests and rank sum tests were used to evaluate the relationship between the ISL1-S629-p immune reaction and the clinicopathological features of the patients. Kaplan-Meier survival curves was generated to evaluate the effect of ISL1-S629-p on patient survival. For all statistical tests, the significance of differences between groups was determined using oneway analysis of variance (ANOVA). Differences were considered statistically significant at $p<0.05$.

\section{Results}

ISL1 interacts with CDK1 in vivo and in vitro

In our previous study, we used mass spectrometry (Lu et al. 2015) to show that ISL1 interacts with CDK1 in MGC803 cell lines (high levels of ISL1 and higher proliferation rates) (Shi et al. 2016). Here, we determined whether ISL1 physically interacts with CDK1/cyclin B1 by using reciprocal co-immunoprecipitation. Our results indicated that these two molecules interact in vivo (Fig. 1a-c). We also used GST linked to ISL1 segments or GST alone to validate the interaction of CDK1 and ISL1 by using a GST pull-down assay; our data suggested that ISL1 interacts directly with CDK1 (Fig. 1d).

\section{CDK1 mediates ISL1 phosphorylation at serine 269 in GC}

To determine whether ISL1 is phosphorylated, we performed co-immunoprecipitation using antibodies against phosphorylated serine and ISL1, and observed phosphorylation serine in ISL1 (Fig. 2a). We then sought to determine which residue in ISL1 could be phosphorylated by CDK1. CDKs often recognize and phosphorylate the serine/threonine-proline $(\mathrm{S} / \mathrm{T}) \operatorname{PX}(\mathrm{R} / \mathrm{K})$ motif (Zhang et al. 
$\mathbf{a}$

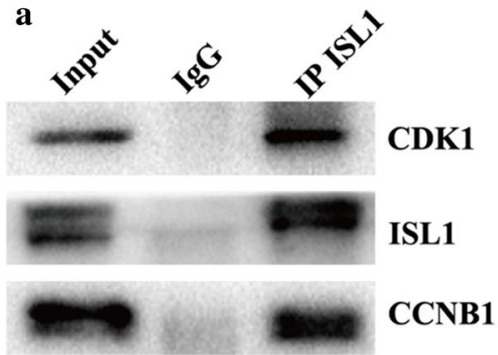

c

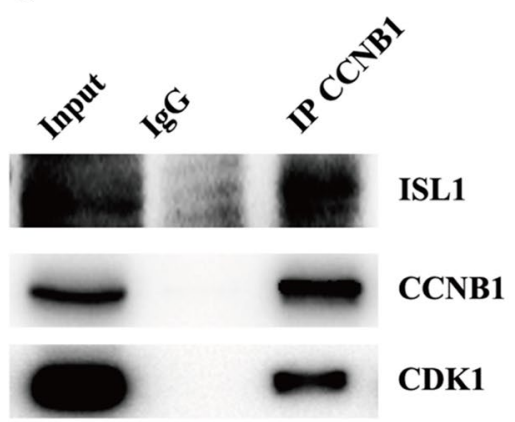

b

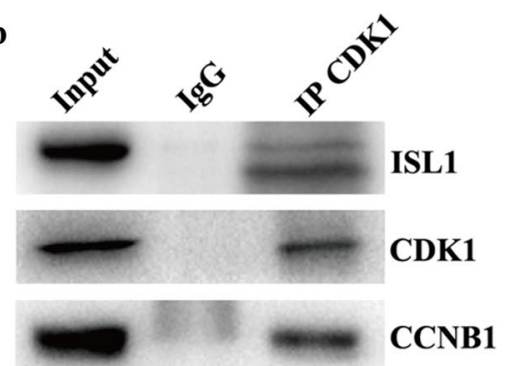

d

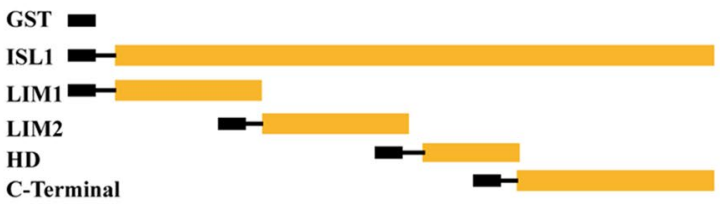

C-Terminal

Fig. 1

ISL1 interacts with CDK1. a-c Co-IP assays were performed to measure the ISL1/CDK1/cyclinB1 complex in MGC803 cells. Lysate was immunoprecipitated with antibodies against ISL1, CDK1, or cyclinB1 and analyzed by immunoblotting. IgG: immunoglobulin G. $\mathbf{d}$ Full-length or truncated ISL1 was used to construct GST-fusion proteins (Top) for pull-down assays with CDK1 protein (Bottom)

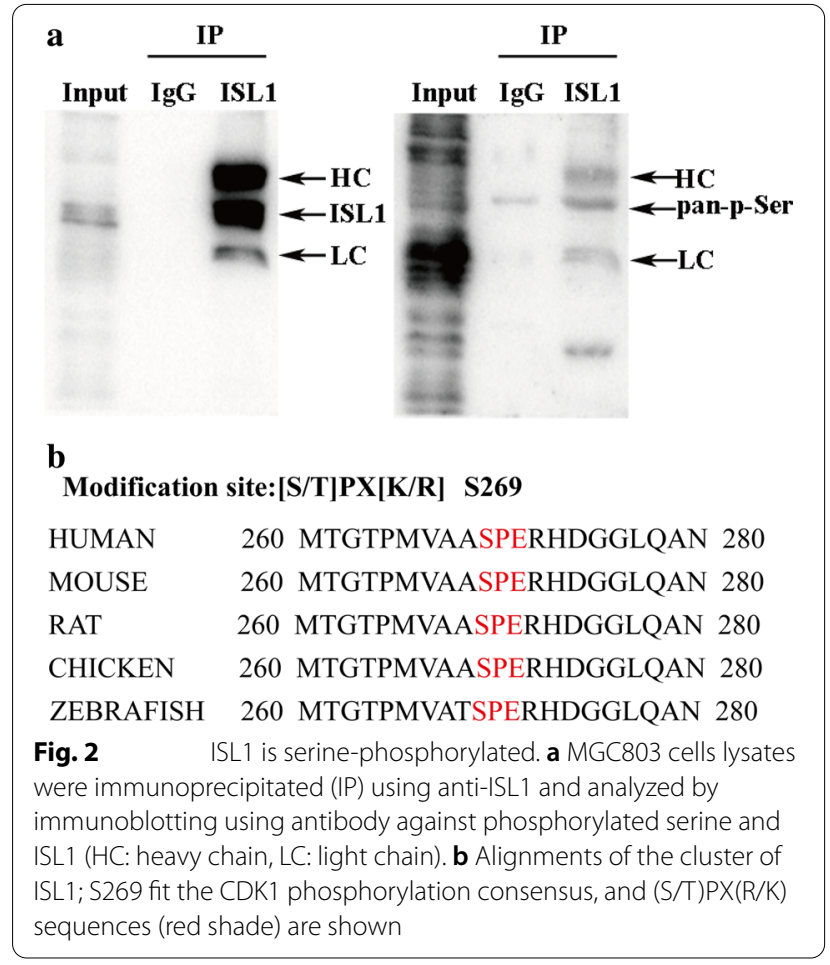

2008). There is only one such motif (S269/P270) present in ISL1 (Fig. 2b), which matched with CDK phosphorylation consensus sites.

Next, we confirmed that CDK1 phosphorylates ISL1 at Ser 269 in vivo. We used a phospho-ISL1-Ser 269 antibody to immunoblot lysates from MGC803 cells transfected with plasmids encoding CDK1 or CDK1 siRNA. The endogenous level of phosphorylated ISL1 at Ser 269 increased when CDK1 was overexpressed, and CDK1 knockdown significantly reduced Ser 269 phosphorylation (Fig. 3a). Similar changes were also observed in the human gastric epithelial cell line GES1 (Additional file 2: Fig. S2). Furthermore, expression of dominant-negative mutant CDK1 (DN-CDK1) also decreased Ser 269 phosphorylation (Fig. 3b). ISL1 was visualized as two bands in the western blots of some samples. These two bands may represent the alternatively spliced variants, ISL1 $\alpha$ and ISL1 $\beta$, which have been reported previously (Ando et al. 2003). These results indicate that Ser 269 is phosphorylated by CDK1 in this cell line.

We replaced the Ser 269 residue with alanine (ISL1S269A), and transfected MKN28 cell lines (which have low levels of ISL1 and proliferate slowly) with wildtype or S269A-mutant ISL1. Phosphorylation of ISL1 was examined by immunoblotting with phospho-specific 


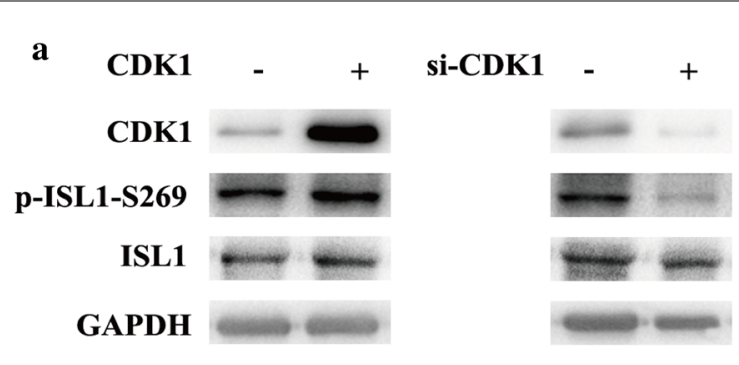

b
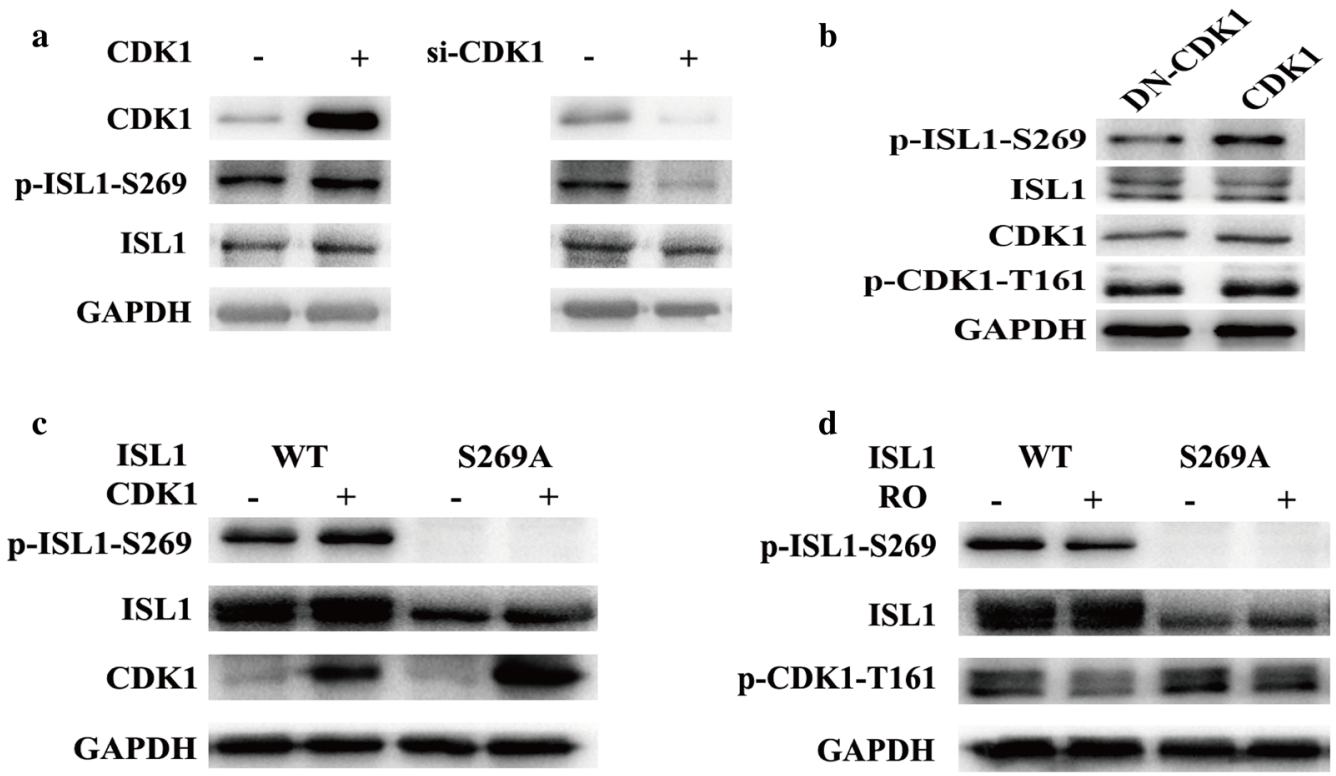

e

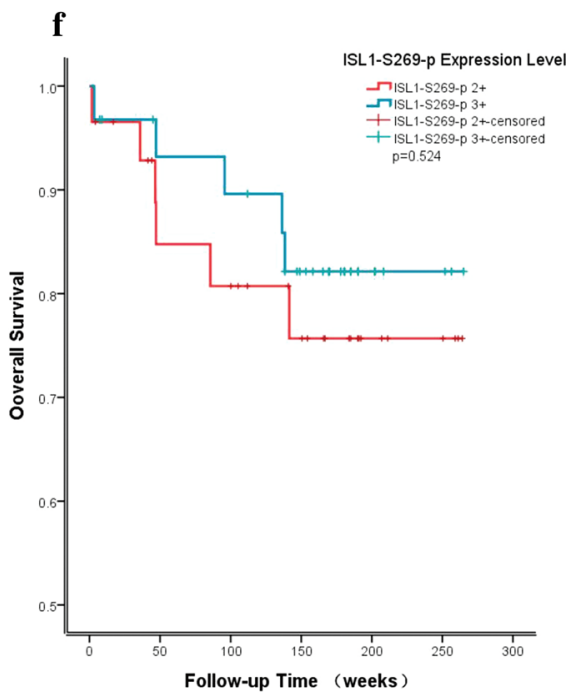

Fig. 3

CDK1 mediates ISL1 phosphorylation at serine 269. a MGC803 cells transfected with plasmid encoding CDK1 or control vector (left), negative control or CDK1 si-RNA (right). Lysate was analyzed by immunoblotting with antibodies against the indicated proteins. b MGC803 cells were transfected with plasmids encoding dominant-negative mutant CDK1 (DN-CDK1) or CDK1, and lysate was analyzed by immunoblotting with antibodies against the indicated proteins. c MKN28 cells expressing wild-type ISL1 or ISL1-S269A were transfected with plasmid encoding CDK1 or control vector. Cell lysates were immunoblotted with antibodies against the indicated proteins. $\mathbf{d}$ MKN28 cells were transfected with plasmids encoding wild-type ISL1 or ISL1-S269A and treated with CDK1 inhibitor RO-3306 or DMSO. Lysates were analyzed by immunoblotting with antibodies against the indicated proteins. GAPDH served as the internal control. e Representative IHC staining of ISL1-S269-p expression in GC (right, $n=60$ ) and paired adjacent tissues (left, $n=60$ ). $\mathbf{f}$ Relationship between expression of ISL1-S629-p and overall survival in gastric cancer

antibody against phospho-S269. Forced expression of CDK1 increased S269 phosphorylation of wild-type ISL1 but not of S269A mutant (Fig. 3c, lane 2 versus lane 4). We also compared the phosphorylation levels of wildtype ISL1 and ISL1 S269A in MKN28 cells transfected with either wild-type (WT) or S269A-mutated ISL1 (S269A), and cultured without or with RO-3306 (10 $\mu \mathrm{M}, 20 \mathrm{~h}$, Additional file 1: Fig. S1), a pan-CDK1 inhibitor (Vassilev et al. 2006). Decreased phosphorylation of Ser 269 was observed in cells expressing WT ISL1 cultured with RO-3306 (Fig. 3d, lane 2), with total ISL1 levels remaining constant. Our results indicated that CDK1 increased phosphorylation of Ser 269, and S269A mutation abolished CDK1-mediated phosphorylation of ISL1. 
In this study, we confirmed the relationship between ISL1-S629-p expression and the overall survival time of patients with GC (35 poorly differentiated adenocarcinoma, 22 moderately differentiated adenocarcinoma, 3 well-differentiated adenocarcinoma) (Fig. 3e). The fiveyear survival rates of patients with ISL1-S629-p ++ and ISL1-629-p+++ were 76 and $82 \%$, respectively. There was no significant correlation between the expression of ISL1-S629-p and overall survival ( $p=0.524)$ (Fig. 3f). We also analyzed the relationship between expression of ISL1-S629-p and clinic pathological parameters of GC. Expression of ISL1-S629-p was not significantly correlated with sex, age, Tumor Node Metastasis (TNM) stages, and distant metastasis, but correlated negatively with the degree of invasion $(p=0.021)$ and differentiation of GC ( $p=0.010)$ (Table 3).

\section{CDK1 phosphorylation of ISL1 promotes ISL1 binding on cyclinB1 and cyclinB2 promoters}

ISL1 functions primarily as a transcription factor. Our next aim was therefore to determine whether the

Table 3 Relationship between expression of ISL1-S629-p and clinic pathological parameters of gastric cancer

\begin{tabular}{|c|c|c|c|}
\hline \multirow[t]{2}{*}{ Characteristic } & \multicolumn{2}{|c|}{ Expression Level of ISL1-S629-p } & \multirow[t]{2}{*}{ p-value } \\
\hline & $\begin{array}{l}++(\%) \\
(n=29)\end{array}$ & $\begin{array}{l}+++(\%) \\
(n=31)\end{array}$ & \\
\hline \multicolumn{4}{|l|}{ Age } \\
\hline$\leq 60$ & $16(55.2 \%)$ & $10(32.3 \%)$ & \multirow[t]{2}{*}{0.073} \\
\hline$>60$ & $13(44.8 \%)$ & $21(67.7 \%)$ & \\
\hline \multicolumn{4}{|l|}{ Gender } \\
\hline Male & $19(65.5 \%)$ & $20(64.5 \%)$ & \multirow[t]{2}{*}{0.935} \\
\hline Female & $10(34.5 \%)$ & $11(35.5 \%)$ & \\
\hline \multicolumn{4}{|c|}{ Depth of invasion } \\
\hline $\mathrm{T} 1-2$ & $12(41.4 \%)$ & $22(71.0 \%)$ & \multirow[t]{2}{*}{0.021} \\
\hline T3-4 & $17(58.6 \%)$ & $9(29.0 \%)$ & \\
\hline \multicolumn{4}{|c|}{ Lymph node metastasis } \\
\hline NO & $10(34.5 \%)$ & $15(48.4 \%)$ & \multirow[t]{2}{*}{0.275} \\
\hline N1-3 & $19(65.5 \%)$ & $16(51.6 \%)$ & \\
\hline \multicolumn{4}{|c|}{ Distant metastasis } \\
\hline MO & $25(86.2 \%)$ & $28(90.3 \%)$ & \multirow[t]{2}{*}{0.702} \\
\hline M1 & $4(13.8 \%)$ & $3(9.7 \%)$ & \\
\hline \multicolumn{4}{|l|}{ TNM stage } \\
\hline 1 & $7(24.1 \%)$ & $14(45.2 \%)$ & \multirow[t]{4}{*}{0.309} \\
\hline$\|$ & $10(34.5 \%)$ & $7(22.6 \%)$ & \\
\hline III & $9(31.0 \%)$ & $6(19.4 \%)$ & \\
\hline IV & $3(10.3 \%)$ & $4(12.9 \%)$ & \\
\hline \multicolumn{4}{|c|}{ Histological grade* } \\
\hline G1 & $1(3.4 \%)$ & $2(6.5 \%)$ & \multirow[t]{3}{*}{0.010} \\
\hline $\mathrm{G} 2$ & $6(20.7 \%)$ & $16(51.6 \%)$ & \\
\hline G3 & 22 (75.9\%) & 13 (41.9\%) & \\
\hline
\end{tabular}

ISL1-CDK1/cyclin B1 complex binds to the promoters of the target genes CCNB1 and CCNB2 in MGC803 cells, by performing a ChIP assay. We found that ISL1-CDK1/cyclin B1 formed a transcriptional activation complex with the $C C N B 1$ and $C C N B 2$ promoters (Fig. 4a, b). Next, we determined whether CDK1-mediated ISL1 phosphorylation affects its transcriptional function. We analyzed ISL1 levels on the promoters of CCNB1 and CCNB2. Quantitative chromatin immunoprecipitation (qChIP) assay results showed lower levels of S269A-mutant ISL1 (S269A) that of wild-type (WT) on the CCNB1 and CCNB2 promoters (Fig. 4c, d). These results suggest that phosphorylation of ISL1 at Ser 269 by CDK1 strengthens ISL1 binding on the $C C N B 1$ and $C C N B 2$ promoters.

\section{Phosphorylation of ISL1 Ser 269 increases transactivation potency and promotes cell proliferation}

We assayed the transactivation potency of wild-type (WT) or S269A-mutant ISL1 (S269A) by expressing each of these proteins in MKN28 cells along with plasmids encoding CDK1 or CDK1 inhibitor RO-3306. As shown in Fig. 5a, b, constitutive expression of either WT or CDK1/WT (Fig. 5a, b; second column versus fourth column) increased transcriptional activity of ISL1 in MKN28 cells. Similarly, S269A or WT/RO-3306 (Fig. 5a, b; third column versus sixth column) decreased the transcriptional activity of ISL1. Importantly, the enhancement of ISL1 by CDK1 was abrogated by the S269A mutation (Fig. 5a, b; fifth column versus seventh column). ISL1 enhances cell proliferation and cell cycle stimulation. We therefore investigated the effect of the S269A mutation on ISL1-mediated biological functions. By using stable transfects expressing wild-type ISL1 and ISL1 S269A in MKN28 cells, we observed significant differences in cell proliferation and cell cycle stimulation. Wild-type ISL1 and ISL1 S269A cells grew faster than did the control cells, and wildtype ISL1 cells grew faster than did ISL1 S269A cells at $48 \mathrm{~h}$ after transfection (Fig. 5c). Moreover, MKN28 cell cycle profiles showed that ISL1 S269A overexpression remarkably decreased the cell population in the $\mathrm{G}_{1}$ phase (from $57.94 \% \pm 1.65$ to $43.15 \% \pm 2.92, p<0.05$ ) and increased the cell population in the $S$ and $G_{2} / M$ phases (from $42.05 \% \pm 1.65$ to $56.84 \% \pm 2.92, p<0.05$ ). This effect was significantly more enhanced by transfection with wild-type ISL1 $\left(\mathrm{G}_{1}\right.$ phase from $57.94 \% \pm$ 1.65 to $36.49 \% \pm 3.48, S$ and $G_{2} / M$ phases from $42.05 \%$ \pm 1.65 to $63.50 \% \pm 3.48, p<0.05$; Fig. $5 \mathrm{~d}$ ). Collectively, our results demonstrated that CDK1-induced phosphorylation of ISL1 Ser 269 is a critical positive regulator of cell proliferation and cell cycle transition. 
a

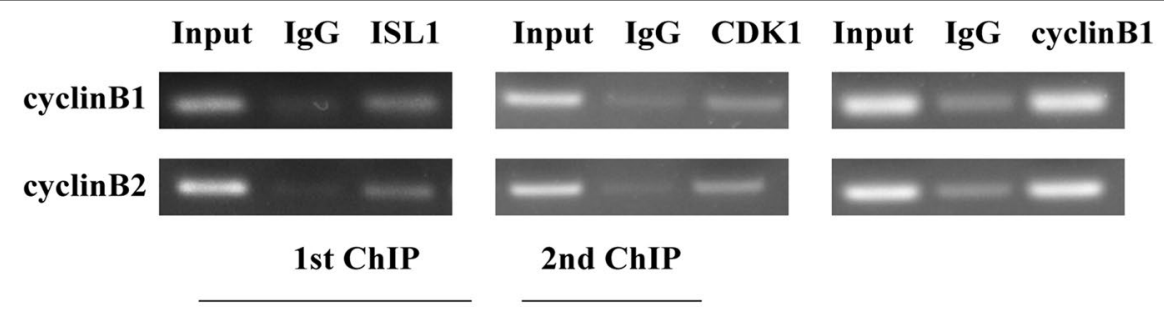

b

Input IgG ISL1 IgG CDK1

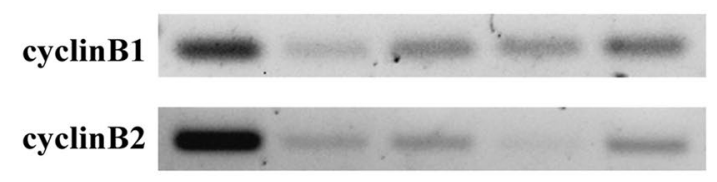

c

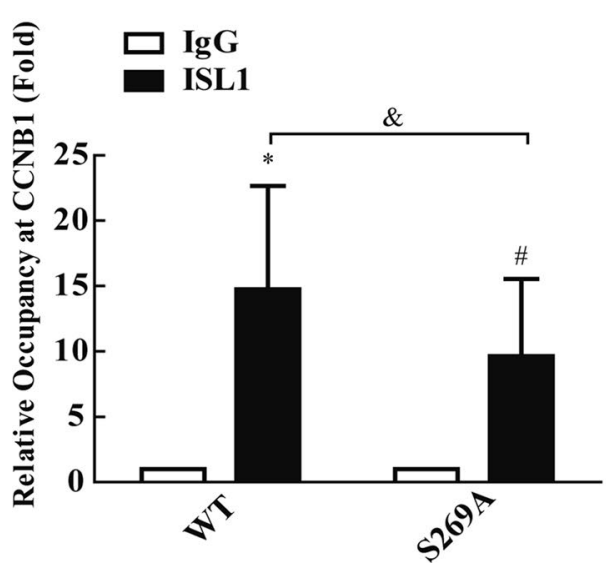

d

Fig. 4 Phosphorylated ISL1 showed improved localization on cyclinB1 and cyclinB2 promoters. a ChIP assay analysis and PCR gel electrophoresis analysis of ISL-1 recruitment to the cyclinB1 and cyclinB2 promoters in MGC803 cells. b ChIP-re-IP assay was performed with anti-ISL-1 or rabbit IgG antibodies and then with anti-CDK1 or lgG antibodies for immunoprecipitation using chromatin harvested from MGC803 cells. c, d Quantitative chromatin immunoprecipitation analysis of cyclinB1 and cyclinB2 promoters in MKN28 cell line transfected with control vector or expressing wild-type ISL1 or ISL1-S269A plasmids. Data are expressed as mean \pm s. d. from three individual experiments. $\left({ }^{*} p<0.05, W T\right.$ vs. control, ${ }^{*} p<0.05$, S269A vs. control, $\$ p<0.05$, WT vs. S269A)

\section{ISL1 phosphorylation increases self-stability but does not increase interaction with CDK1}

To assess whether ISL1 phosphorylation mediates selfstability, NIH3T3 cells were transfected with wild-type ISL1 or ISL1 S269A for $48 \mathrm{~h}$ and treated with cycloheximide $(\mathrm{CHX})$ to block protein synthesis for various lengths of time. Total cell lysates were prepared and immunoblotted. ISL1 S269A protein levels decreased within $6 \mathrm{~h}$, and further reduced $10 \mathrm{~h}$ after CHX treatment, while wild-type ISL1 protein levels remained unchanged within $8 \mathrm{~h}$ after treatment with $\mathrm{CHX}$ (Fig. 6a). We then studied the effect of CDK1 modification on ISL1-CDK1 combination. Nuclear extracts prepared from NIH3T3 cells transfected with CDK1 and either ISL1-WT, or ISL1-S269A for $48 \mathrm{~h}$ were subjected to Co-IP assay. We did not observe significant differences in ISL1-CDK1 binding (Fig. 6b). These data suggest that
ISL1 phosphorylation increases self-stability but does not increase its interaction with CDK1.

\section{Discussion}

The expression and DNA methylation of ISL1 in carcinoma has been studied in many tumors (Dong et al. 1991; Erlenbach-Wunsch et al. 2014; Graham et al. 2013; Kim et al. 2013; Kitchen et al. 2015; Li et al. 2018; Wang et al. 2014; Zhang et al. 2014). We demonstrated previously that ISL1 expression may serve as a specific marker for GC treatment and prognosis (Guo et al. 2015). Further, ISL1 enhanced tumor growth and targeted the downstream genes CCNB1, CCNB2, and C-myc in GC (Shi et al. 2016). ISL1 was also positively associated with poor outcome in primary GC (Guo et al. 2019). However, the role of ISL1 in GC proliferation and carcinogenesis, and 
a

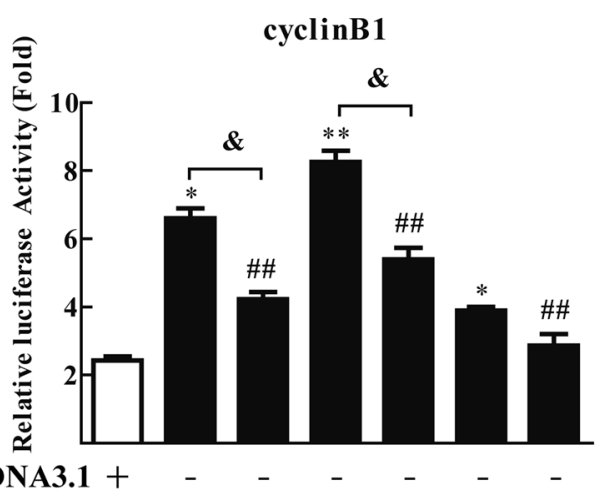

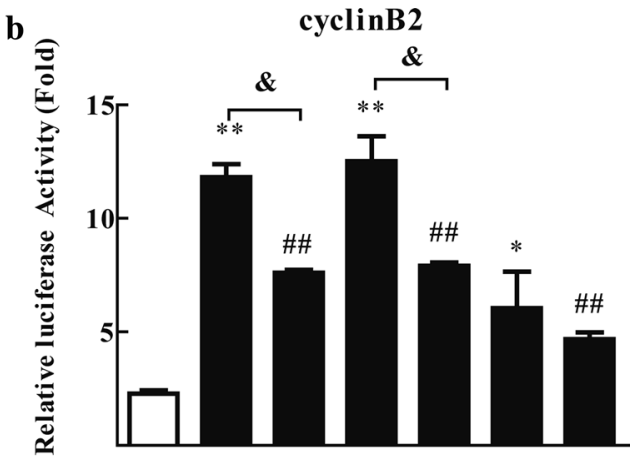

$$
\begin{array}{rccccccr}
\text { pcDNA3.1 + } & - & - & - & - & - & - & \text { pcDNA3.1 + } \\
\text { ISL1-WT - } & + & - & + & - & + & - & \text { ISL1-WT - } \\
\text { ISL1-S269A - } & - & + & - & + & - & + \\
\text { ISL1-S269A - } \\
\text { CDK1 - } & - & - & + & + & - & - & \text { CDK1 - } \\
\text { RO-3306 - } & - & - & - & - & + & + & \text { RO-3306 - }
\end{array}
$$
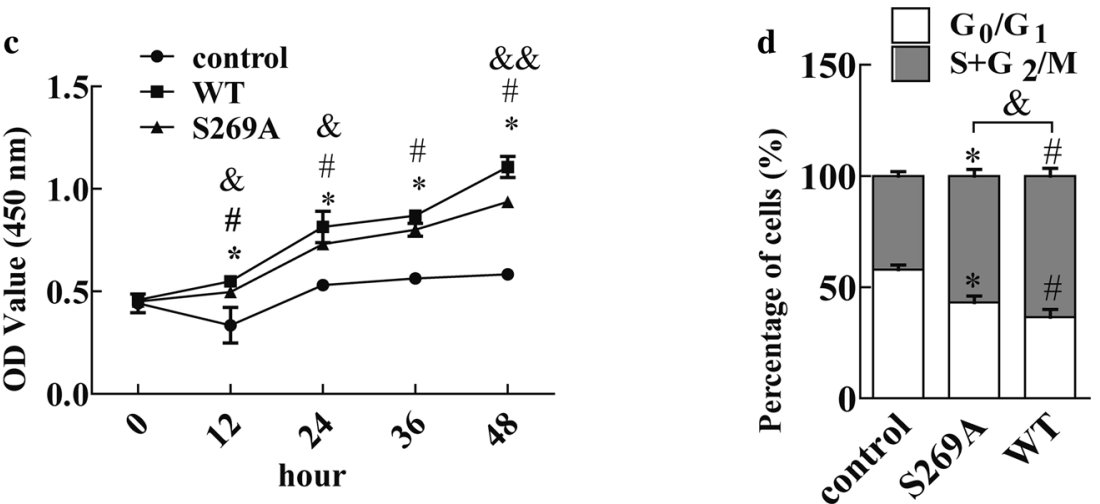

Fig. 5

Phosphorylation Ser 269 of ISL1 increases transactivation potency. $\mathbf{a}, \mathbf{b}$ Effect of CDK1 activity on the transcriptional activity of wild-type ISL1 or S269A ISL1. Transcriptional activity of wild-type ISL1 and S269A ISL1 on cyclinB1-luc and cyclinB2-luc determined by luciferase reporter assay in MKN28 cells. c Cell proliferation was studied by CCK-8 analysis of MKN28 cells transfected with plasmids encoding control vector and wild-type ISL1 or ISL1-S269A. d Cell cycle distributions were analyzed by flow cytometry in MKN28 transfected with plasmids encoding control vector and wild-type ISL1 or ISL1-S269A. The data represent 3 independent experiments, each performed in triplicate. Each bar represents mean \pm SD. $\left({ }^{*} p<0.05\right.$, WT vs. control, ${ }^{\#} p<0.05$, S269A vs. control, ${ }^{\$} p<0.05$, WT vs. S269A)

the mechanism by which ISL1 regulates proliferation and tumorigenesis remains unclear.

In this study, we found evidence that ISL1 interacts with CDK1 in vivo and in vitro. We first confirmed ISL1 interaction with CDK1 in MGC803 cells (which showed high levels of ISL1 and proliferated more quickly) by mass spectrometry. We then determined that ISL1 interacts with CDK1/cyclin and confirmed the interaction by using a GST pull-down assay to show that ISL1 interacts directly with CDK1.

Next, we demonstrated that CDK1 specifically phosphorylates ISL1 at S269, thereby promoting the transcriptional activity of ISL1. CDK1 is a serine/threonine kinase that is often dysregulated in human cancers including GC (Gao et al. 2014; Lee et al. 2016). The ISL1 C-terminal region also contains the LIM homeobox 3 (Lhx3)-binding domain (LBD), which is a candidate intramolecular interaction domain (Gadd et al. 2013). In addition, S269 in ISL1 fits the cyclin dependent kinase 1 (CDK1) phosphorylation consensus, $(\mathrm{S} / \mathrm{T}) \mathrm{PX}(\mathrm{R} / \mathrm{K})$, and is the only such motif (S269/P270) present in ISL1, although mass spectrometry analysis is required to determine the phosphorylation status of Ser269 residue in ISL1 in vitro. We then used the phospho-ISL1-Ser 269 antibody for immunoblot analysis of the lysates from MGC803 cells transfected with plasmids encoding CDK1, CDK1 siRNA, CDK1 (DN-CDK1), or CDK1 inhibitors. Our results indicated that Ser 269 is phosphorylated in this cell line. At the same time, we also tried to observe whether CDK1 phosphorylated ISL1 in normal human mucosal epithelial 


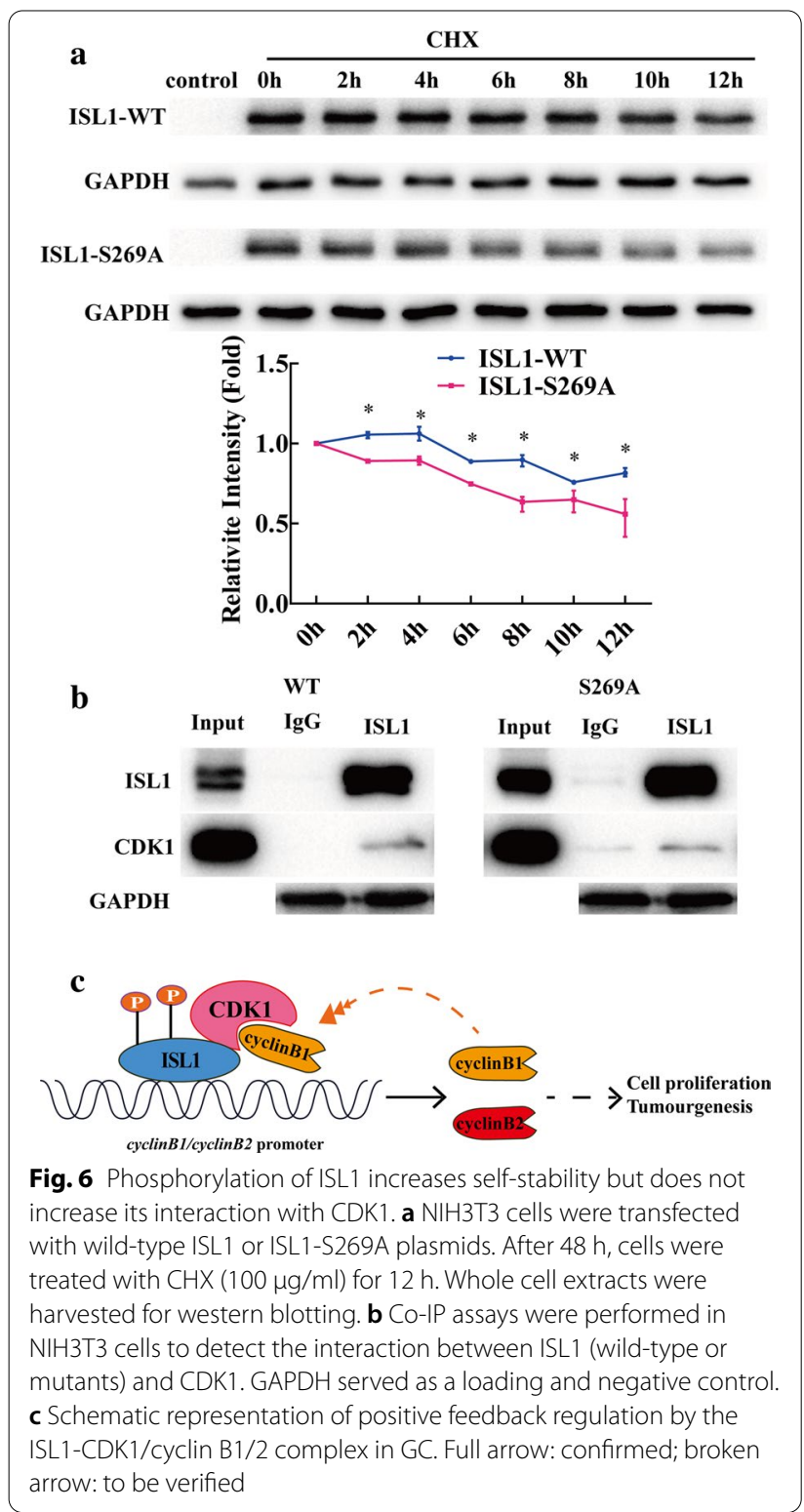

cells GES1, and it was found that the overexpression or knockdown of CDK1 greatly affected the expression of ISL1 protein (Additional file 2: Fig. S2). This may be due to the low expression of ISL1 in GES1 and the regulation of phosphorylation is different from tumor cells. We then replaced the Ser 269 residue with alanine (ISL1S269A), transfected MKN28 cells (which had low levels of ISL1 and proliferated more slowly) with wild-type or S269Amutated ISL1. CDK1 increased phosphorylation of the wild-type ISL1 but not of the S269A mutant. Because ISL1 functions primarily as a transcription factor and forms a complex to regulate target genes (Li et al. 2014; Li et al. 2014a, b). We determined whether the ISL1-CDK1/ cyclin B1 complex binds to the promoters of the target genes $C C N B 1$ and $C C N B 2$. ChIP and quantitative chromatin immunoprecipitation (qChIP) assays showed that the ISL1-CDK1/cyclin B1 complex showed stronger localization on $C C N B 1$ and $C C N B 2$ promoters. This may result in positive feedback regulation by the ISL1CDK1/cyclin B1/2 complex in GC (Fig. 6c), although this hypothesis remains to be investigated.

We further demonstrated that CDK1-mediated modification of ISL1 may contribute to tumorigenesis and proliferation via regulation of downstream genes in GC (Benanti 2016). Up- or downregulation of ISL1 in GC cells promoted cell cycle transition into the S/G2/M phases or cell cycle arrest in the G0/G1 phases respectively. More importantly, ISL1 regulated $C C N B 1$ and $C C N B 2$, and consequently influenced the cell cycle. We transfected wild-type (WT) or S269A-mutated ISL1 (S269A) into MKN28 cells along with plasmids encoding CDK1 or CDK1 inhibitor RO-3306. The WT or CDK1/ WT cells showed increased transcriptional activity of ISL1. Similarly, S269A or WT/RO-3306 decreased the transcriptional activity of ISL1. Importantly, the stimulatory effect of CDK1 on ISL1 was abrogated by the S269A mutation. By using stable transfects expressing wild-type ISL1 and ISL1 S269A in MKN28 cells, we observed that wild-type ISL1 cells grew faster than did ISL1 S269A cells. Consistently, the MKN28 cell cycle profiles demonstrated that ISL1 S269A overexpression remarkably decreased the cell population in the G1 phase and increased the cell population in the $\mathrm{S}$ and $\mathrm{G} 2 / \mathrm{M}$ phases. Importantly, this effect was significantly more increased by transfection with wild-type ISL1. We demonstrated that phosphorylation of Ser-269 strengthens the ability of ISL1 to activate transcription and stimulate proliferation. Thus, ISL1 activity is likely linked with the cell cycle via its regulation by cyclin-dependent kinase 1 , through phosphorylation of distinct sites. These results indicate for the first time that the phosphorylation status of ISL1 is essential for sustained activation of ISL1 to promote cell proliferation via regulation of downstream genes. However, detection of the mechanism by which ISL1 is activated and targeted for degradation remain to be studied. Furthermore, the potential involvement of other molecules involved in the modification of ISL1 by CDK1 remains to be clarified.

\section{Conclusions}

We demonstrated that ISL1 interacts with CDK1, which phosphorylates ISL1 at Ser-269. ISL1-CDK1 showed improved binding on cyclin B1 and cyclin B2 promoters, and the phosphorylation increased ISL1 transcriptional activity and self-stability. It can be speculated that sustained activation of ISL1 promotes cell proliferation 
and tumorigenesis via a positive feedback mechanism (Fig. 6c). These findings provide, to our knowledge, the first evidence that CDK1 phosphorylates ISL1 Ser-269, and could provide a theoretical basis for the development of novel strategies and targets for GC treatment.

\section{Supplementary Information}

The online version contains supplementary material available at https://doi. org/10.1186/s10020-021-00302-6.

Additional file 1: Fig. S1 RO-3306 arrests cells at the $\mathrm{G}_{2} / \mathrm{M}$ phase border. Cell cycle profile of proliferating MGC803 (A) or treated with RO-3306 $(9 \mu \mathrm{M})$ for $20 \mathrm{~h}(\mathrm{~B})$

Additional file 2: Fig. S2. GES1 cells transfected with plasmid encoding CDK1 or control vector, negative control or CDK1 si-RNA. Lysate was analyzed by immunoblotting with antibodies against the indicated proteins

\section{Acknowledgements}

We appreciate all participants in this work.

\section{Authors' contributions}

NXM and LM performed the experiments and prepared the manuscript; XQS and ZQ analyzed the data, DY performed the IHC analysis and cell cultures; SQ and WWP designed the experiments, participated in discussions and polished the manuscript. All authors read and approved the final manuscript.

\section{Funding}

This work was supported by the National Natural Science Foundation of China (No. 81,760,426, 81,370,236, 31,960,145); Yunnan Science and Technology Department Fund (2018FE001(-314)), (2018FE001(-315)).

\section{Declarations}

\section{Ethical approval and consent to participate}

This research was performed in accordance with amended Declaration of Helsinki, with the approval of the Kunming Medical University Research Ethics Committee

\section{Consent for publication}

All authors give consent for publication.

\section{Competing interests}

The authors declare no conflicts of interest.

\begin{abstract}
Author details
${ }^{1}$ Clinical Laboratory, The Third Affiliated Hospital, Kunming Medical University \&Yunnan Cancer Center, Yunnan, Kunming, P. R. China. ${ }^{2}$ Pathology Department, The Third Affiliated Hospital, Kunming Medical University \& Yunnan Cancer Center, Yunnan, Kunming, P. R. China. ${ }^{3}$ Department of Biochemistry and Molecular Biology, School of Basic Medical Sciences, Kunming Medical University, Yunnan, Kunming, P. R. China. ${ }^{4}$ Department of Biochemistry and Molecular Biology, School of Basic Medical Sciences, Beijing Key Laboratory of Protein Posttranslational Modifications and Cell Function, Key Laboratory of Molecular Cardiovascular Sciences, Ministry of Education of China, Peking University, Beijing, P. R. China.
\end{abstract}

Received: 31 December 2020 Accepted: 14 April 2021

Published online: 07 May 2021

\section{References}

Ando K, Shioda S, Handa H, Kataoka K. Isolation and characterization of an alternatively spliced variant of transcription factor Islet-1. J Mol Endocrinol. 2003;31:419-25.
Benanti JA. Create, activate, destroy, repeat: Cdk1 controls proliferation by limiting transcription factor activity. Curr Genet. 2016;62:271-6.

Bu L, Jiang X, Martin-Puig S, Caron L, Zhu S, Shao Y, Roberts DJ, Huang PL, Domian IJ, Chien KR. Human ISL1 heart progenitors generate diverse multipotent cardiovascular cell lineages. Nature. 2009;460:113-7.

$\mathrm{Bu}$ Z, Ji J. A current view of gastric cancer in China. Translational Gastrointestinal Cancer. 2013:1-4.

Dong J, Asa SL, Drucker DJ. Islet cell and extrapancreatic expression of the LIM domain homeobox gene isl-1. Mol Endocrinol. 1991;5:1633-41.

Erlenbach-Wunsch K, Haller F, Taubert H, Wurl P, Hartmann A, Agaimy A. Expression of the LIM homeobox domain transcription factor ISL1 (Islet-1) is frequent in rhabdomyosarcoma but very limited in other soft tissue sarcoma types. Pathology. 2014;46:289-95.

Ferlay J, Soerjomataram I, Dikshit R, Eser S, Mathers C, Rebelo M, Parkin DM, Forman D, Bray F. Cancer incidence and mortality worldwide: sources, methods and major patterns in GLOBOCAN 2012. Int J Cancer. 2015;136:E359-86.

Gadd MS, Jacques DA, Nisevic I, Craig VJ, Kwan AH, Guss JM, Matthews JM. A structural basis for the regulation of the LIM-homeodomain protein islet 1 (Is|1) by intra- and intermolecular interactions. J Biol Chem. 2013;288:21924-35

Gao SY, Li J, Qu XY, Zhu N, Ji YB. Downregulation of Cdk1 and cyclinB1 expression contributes to oridonin-induced cell cycle arrest at G2/M phase and growth inhibition in SGC-7901 gastric cancer cells. Asian Pac J Cancer Prev. 2014;15:6437-41.

Graham RP, Shrestha B, Caron BL, Smyrk TC, Grogg KL, Lloyd RV, Zhang L. Islet-1 is a sensitive but not entirely specific marker for pancreatic neuroendocrine neoplasms and their metastases. Am J Surg Pathol. 2013;37:399-405.

Guo T, Wang W, Zhang H, Liu Y, Chen P, Ma K, Zhou C. ISL1 promotes pancreatic islet cell proliferation. PLoS One. 2011;6:e22387.

Guo C, Wang W, Shi Q, Chen P, Zhou C. An abnormally high expression of ISL-1 represents a potential prognostic factor in gastric cancer. Hum Pathol. 2015;46:1282-9.

Guo T, Wen XZ, Li ZY, Han HB, Zhang CG, Bai YH, Xing XF, Cheng XJ, Du H, Hu $Y$, et al. ISL1 predicts poor outcomes for patients with gastric cancer and drives tumor progression through binding to the ZEB1 promoter together with SETD7. Cell Death Dis. 2019;10:33.

Karlsson O, Thor S, Norberg T, Ohlsson H, Edlund T. Insulin gene enhancer binding protein $|s|-1$ is a member of a novel class of proteins containing both a homeo- and a Cys-His domain. Nature. 1990;344:879-82.

Kim YJ, Yoon HY, Kim JS, Kang HW, Min BD, Kim SK, Ha YS, Kim IY, Ryu KH, Lee SC, et al. HOXA9, ISL1 and ALDH1A3 methylation patterns as prognostic markers for nonmuscle invasive bladder cancer: array-based DNA methylation and expression profiling. Int J Cancer. 2013;133:1135-42.

Kim E, Jiang M, Huang H, Zhang Y, Tjota N, Gao X, Robert J, Gilmore N, Gan L, Que J. Isl1 Regulation of Nkx2.1 in the Early Foregut Epithelium Is Required for Trachea-Esophageal Separation and Lung Lobation. Dev Cell. 2019;51:675-83 e674.

Kitchen MO, Bryan RT, Haworth KE, Emes RD, Luscombe C, Gommersall L, Cheng KK, Zeegers MP, James ND, Devall AJ, et al. Methylation of HOXA9 and ISL1 Predicts Patient Outcome in High-Grade Non-Invasive Bladder Cancer. PLoS One. 2015;10:e0137003.

Lee MH, Cho Y, Kim DH, Woo HJ, Yang JY, Kwon HJ, Yeon MJ, Park M, Kim SH, Moon C, et al. Menadione induces G2/M arrest in gastric cancer cells by down-regulation of CDC25C and proteasome mediated degradation of CDK1 and cyclin B1. Am J Transl Res. 2016;8:5246-55.

Li R, Wu F, Ruonala R, Sapkota D, Hu Z, Mu X. Isl1 and Pou4f2 form a complex to regulate target genes in developing retinal ganglion cells. PLoS One. 2014a;9:e92105.

Li Y, Pan J, Wei C, Chen J, Liu Y, Liu J, Zhang X, Evans SM, Cui Y, Cui S. LIM homeodomain transcription factor Is| 1 directs normal pyloric development by targeting Gata3. BMC Biol. 2014b;12:25.

Li L, Sun F, Chen X, Zhang M. ISL1 is upregulated in breast cancer and promotes cell proliferation, invasion, and angiogenesis. Onco Targets Ther. 2018;11:781-9.

Liu Z, Li T, Liu Y, Jia Z, Li Y, Zhang C, Chen P, Ma K, Affara N, Zhou C. WNT signaling promotes $\mathrm{Nkx2}$. 5 expression and early cardiomyogenesis via downregulation of Hdac1. Biochimica et Biophysica Acta (BBA)-Molecular Cell Research. 2009; 1793:300-311. 
Liu Y, Li Y, Li T, Lu H, Jia Z, Wang W, Chen P, Ma K, Zhou C. POU homeodomain protein OCT1 modulates islet 1 expression during cardiac differentiation of P19CL6 cells. Cell Mol Life Sci. 2011;68:1969-82.

Lu D, Liu L, Ji X, Gao Y, Chen X, Liu Y, Liu Y, Zhao X, Li Y, Li Y, et al. The phosphatase DUSP2 controls the activity of the transcription activator STAT3 and regulates TH17 differentiation. Nat Immunol. 2015;16:1263-73.

McDonald IM, Grant GD, East MP, Gilbert TS, Wilkerson EM, Goldfarb D, Beri J Herring LE, Vaziri C, Cook JG, et al. Mass spectrometry-based selectivity profiling identifies a highly selective inhibitor of the kinase MELK that delays mitotic entry in cancer cells. J Biol Chem. 2020. https://doi.org/10. 1074/jbc.RA119.011083.

Mertins P, Qiao JW, Patel J, Udeshi ND, Clauser KR, Mani DR, Burgess MW, Gillette MA, Jaffe JD, Carr SA. Integrated proteomic analysis of post-translational modifications by serial enrichment. Nat Methods. 2013;10:634-7.

Sharma K, D'Souza RC, Tyanova S, Schaab C, Wisniewski JR, Cox J, Mann M. Ultradeep human phosphoproteome reveals a distinct regulatory nature of Tyr and Ser/Thr-based signaling. Cell Rep. 2014;8:1583-94.

Shi Y, Zhao S, Li J, Mao B. Islet-1 is required for ventral neuron survival in Xenopus. Biochem Biophys Res Commun. 2009;388:506-10.

Shi Q, Wang W, Jia Z, Chen P, Ma K, Zhou C. ISL1, a novel regulator of CCNB1, CCNB2 and c-MYC genes, promotes gastric cancer cell proliferation and tumor growth. Oncotarget. 2016;7:36489-500.

Vassilev LT, Tovar C, Chen S, Knezevic D, Zhao X, Sun H, Heimbrook DC, Chen L. Selective small-molecule inhibitor reveals critical mitotic functions of human CDK1. Proc Natl Acad Sci U S A. 2006;103:10660-5.

Waddell T, Chau I, Cunningham D, Gonzalez D, Okines AF, Okines C, Wotherspoon A, Saffery C, Middleton G, Wadsley J, et al. Epirubicin, oxaliplatin, and capecitabine with or without panitumumab for patients with previously untreated advanced oesophagogastric cancer (REAL3): a randomised, open-label phase 3 trial. Lancet Oncol. 2013;14:481-9.
Wang X, He C, Hu X. LIM homeobox transcription factors, a novel subfamily which plays an important role in cancer (Review). Oncol Rep. 2014:31:1975-85.

Wang Y, Li Y, Guo C, Lu Q, Wang W, Jia Z, Chen P, Ma K, Reinberg D, Zhou C. ISL1 and JMJD3 synergistically control cardiac differentiation of embryonic stem cells. Nucleic Acids Res. 2016;44:6741-55.

Zahedi RP, Lewandrowski U, Wiesner J, Wortelkamp S, Moebius J, Schutz C, Walter U, Gambaryan S, Sickmann A. Phosphoproteome of resting human platelets. J Proteome Res. 2008;7:526-34.

Zhang Y, Zhang H, Liang J, Yu W, Shang Y. SIP, a novel ankyrin repeat containing protein, sequesters steroid receptor coactivators in the cytoplasm. EMBO J. 2007;26:2645-57.

Zhang L, Fried FB, Guo H, Friedman AD. Cyclin-dependent kinase phosphorylation of RUNX1/AML1 on 3 sites increases transactivation potency and stimulates cell proliferation. Blood. 2008;111:1193-200.

Zhang H, Wang WP, Guo T, Yang JC, Chen P, Ma KT, Guan YF, Zhou CY. The LIM-homeodomain protein ISL1 activates insulin gene promoter directly through synergy with BETA2. J Mol Biol. 2009:392:566-77.

Zhang Q, Yang Z, Jia Z, Liu C, Guo C, Lu H, Chen P, Ma K, Wang W, Zhou C. ISL-1 is overexpressed in non-Hodgkin lymphoma and promotes lymphoma cell proliferation by forming a p-STAT3/pc-Jun/ISL-1 complex. Mol Cancer. 2014;13:181.

Zhang Q, Huang R, Ye Y, Guo X, Lu J, Zhu F, Gong X, Zhang Q, Yan J, Luo L, et al. Temporal requirements for ISL1 in sympathetic neuron proliferation, differentiation, and diversification. Cell Death Dis. 2018:9:247.

\section{Publisher's note}

Springer Nature remains neutral with regard to jurisdictional claims in published maps and institutional affiliations.
Ready to submit your research? Choose BMC and benefit from:

- fast, convenient online submission

- thorough peer review by experienced researchers in your field

- rapid publication on acceptance

- support for research data, including large and complex data types

- gold Open Access which fosters wider collaboration and increased citations

- maximum visibility for your research: over $100 \mathrm{M}$ website views per year

At $\mathrm{BMC}$, research is always in progress.

Learn more biomedcentral.com/submissions 